Www.jmscr.igmpublication.org

ISSN (e)-2347-176x ISSN (p) 2455-0450

crossrefDOI: https://dx.doi.org/10.18535/jmscr/v7i1.103

\title{
Effect of antioxidant rich diet in patients suffering from osteoarthritis knee
}

\author{
Author \\ Dr Nancy Sharma \\ ASCOMS Hospital
}

\begin{abstract}
The study was conducted at physiotherapy department, ASCOMS Hospital, Sidhra where 50 subjects suffering from knee osteoarthritis were taken to determine the relationship between antioxidant rich diet and osteoarthritis knee. Inclusion criteria consisted of female patient with ages between 21 to 80 years old. Patients having knee osteoarthritis were physically examined before and after the study and results were compared thereof to rule out the significant association between antioxidant rich diet and knee osteoarthritis.
\end{abstract}

\section{Introduction}

\section{Anatomy}

Knee joint is a synovial hinge joint formed between femur, tibia and patella. A thin layer of hyaline cartilage is present between the joint surfaces that protect the underlying bone from damage. Between these bones, meniscus is present which acts as a shock absorber during strenuous activities like jumping and running. A joint capsule surrounds the bones of the knee to provide strength \& lubrication. Synovial fluid is present in the hollow space between the bones, thereby lubricating the knee to reduce friction \& wear.

\section{Kinesiology}

Viewed in the sagittal plane, femur's articulating surface is convex while tibia is concave. Knee kinematics is based on the rules of concavity \& convexity \& is described in terms of open \& closed chain kinematics. Also, the screw home mechanism considered to be a very key element to knee stability which is the rotation between tibia
$\&$ femur which occurs at the end of knee extension.

\section{Pathology}

Osteoarthritis is a condition in which the natural cushion between the joint cartilages wears away results in rubbing of joint surfaces. This cause pain, swelling, stiffness, decrease ability to move and formation of bone spurs. Condition is more common in women and elderly.

\section{Antioxidant Diet \& Osteoarthritis}

Usually the knee gets inflamed during wear and tear mechanism. Inflammation produces free radicals, the cell damaging molecules that are formed in response to toxins and natural body processes. The synovium is more prone to free radical. Antioxidants protect the body from free radicals. Food rich in antioxidants such as omega3 helps to reduce two protein called $\mathrm{C}$ - reactive protein and IL-6, that cause inflammation of the knee joint. Honey is also a good source of 
antioxidant and is rich in phenolic acids and flavonoids. Turmeric contains curumin which has powerful anti inflammatory effects and is a very strong antioxidant and it requires black pepper to enhance its absorption. Other sources of antioxidants are garlic, walnuts, flax seeds, chia seeds, tea, green tea, dark chocolate, coffee, vit A rich foods like carrots, broccoli, peaches, apricots, olive oil, and citrus etc

\section{Methodology}

In osteoarthritis initiative, 50 subjects with diagnosed knee osteoarthritis were taken. Methodology involves data collection through questionnaire, interview and physical assessment. Dietary recall, food frequency and food charts were used to gather dietary information and to monitor intake. The patients were physically assessed and examined for pain, swelling, stiffness, mobility, gait pattern and were recorded in a tabular form. The patients were put on an antioxidant rich diet for over three months. After this period of diet regime, same physical parameters were recorded and comparative study was done to rule out the effectiveness of diet in relieving the symptoms of knee osteoarthritis.

\section{Results}

\begin{tabular}{|c|c|}
\hline $\begin{array}{c}\text { AGE GROUP } \\
(21-70 \text { yrs })\end{array}$ & NO. OF SUBJECTS \\
\hline $21-30$ & $(50)$ \\
\hline $31-40$ & 2 \\
\hline $41-50$ & 2 \\
\hline $51-60$ & 8 \\
\hline $61-70$ & 20 \\
\hline & 18 \\
\hline
\end{tabular}

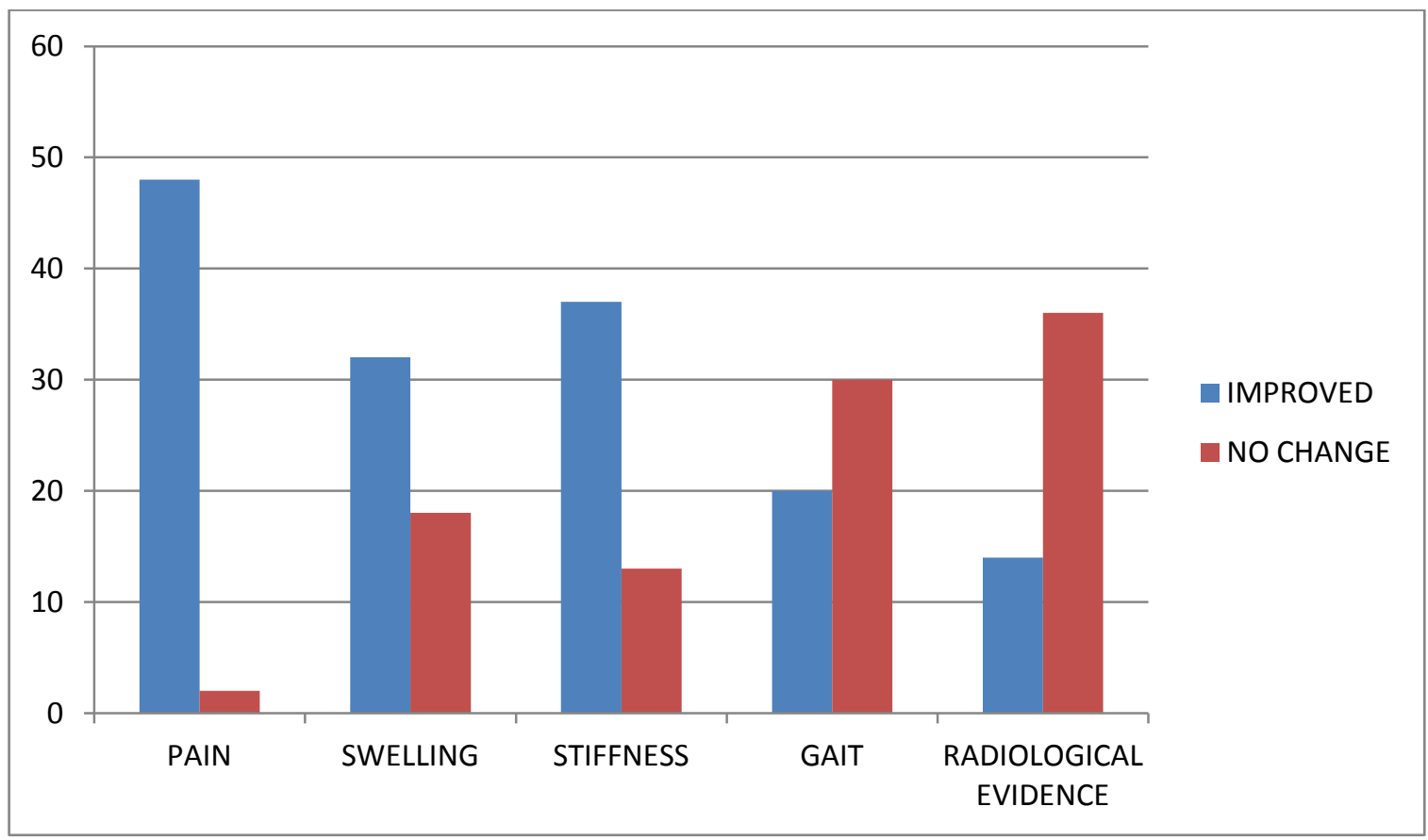

\section{Conclusion}

The study showed that there is positive correlation between antioxidant rich diet and relieve of knee pain in women. But the obese women who lost their weight during the study showed more positive outcome of the study.

\section{References}

1. Miranda H, Vilkari - Juntura E, Martikarinen R, Riihimatii H. A prospective study on knee pain $\&$ its risk factors. Osteoarthritis cartilage 2002

2. Messiee SP, Loeser RF, Miller GD, Moigam TM, Rejestic WJ, Sevick MA, et al. Exercise and dietary weight loss in overweight and obese adults with knee osteoarthritis: the Arthritis, Diet and activity promotion trial. Arthritis Rheum 2004 
3. Sutipornphalangual W, et al. J, Med Assoc Thai. Free radicals in primary knee osteoarthritis, 2009.

4. Ashraf JM, et al. ECCI J. Biochemical and immunological parameters as indicators of OA Subject: role of $\mathrm{OH}$ - collagen in auto antibodies generation, 2015

5. Gerritsen M.E. Carley W.W, Ranges G.E, et al. Flavonoids inhibit cytokine induced endothelial cell adhesion protein gene expression. The American journal of pathology. 1995

6. Machin LJ, Bendict, A; Free radical tissue damage: protective role of antioxidant nutrients. FFASEB J. 1987

7. Conroier T, Mathera P, Bonjean M.A complex of three natural anti inflammatory agents provides relief for osteoarthritis pain. Altren Ther Health Med. 2014

8. Felson DT, Anthony JM, Narmark A, Anderson JJ. Weight loss reduces the risk for symptomatic knee osteoarthritis in women. The Framingham study. Ann intern Med 1992. 